Human Mutation 2020; 41 (10): 1738-1744 (DOI: 10.1002/humu.24075)

The expanding clinical phenotype of germline $A B L 1$-associated congenital heart defects

\title{
and skeletal malformations syndrome
}

Chun-An Chen ${ }^{1,2}$, Emeline Crutcher ${ }^{1,2,3}$, Harinder Gill ${ }^{4,5}$, Tanya N. Nelson ${ }^{4,6,7}$, Laurie A.

Robak $^{1,2,8}$, Marjolijn C. J. Jongmans ${ }^{9,+}$, Rolph Pfundt $^{9}$, Chitra Prasad ${ }^{10,11}$, Roberta A.

Berard $^{10,11,12}$, Madeleine Fannemel ${ }^{13}$, Eirik Frengen ${ }^{14}$, Doriana Misceo ${ }^{14}$, Keri Ramsey $^{15}$,

CAUSES Study ${ }^{4}$, C4RCD Research Group ${ }^{15}$, Yaping Yang ${ }^{1,16,17}$, Christian P. Schaaf ${ }^{1,2,18,19^{*}}$,

and Xia Wang $1,16,17,19^{*}$

${ }^{1}$ Department of Human and Molecular Genetics, Baylor College of Medicine, Houston, TX, USA.

'Jan and Dan Duncan Neurological Research Institute, Texas Children's Hospital, Houston, TX, USA.

${ }^{3}$ Development, Disease Models \& Therapeutics Graduate Program, Baylor College of Medicine, Houston, TX, USA.

${ }^{4}$ Department of Medical Genetics, University of British Columbia, Vancouver, BC, Canada.

${ }^{5}$ Provincial Medical Genetics Program, BC Women's Hospital and Health Centre, Vancouver, BC,

Canada.

${ }^{6}$ Department of Pathology and Laboratory Medicine, BC Children's Hospital, Vancouver, BC,

Canada.

${ }^{7}$ Department of Pathology and Laboratory Medicine, University of British Columbia, Vancouver,

BC, Canada.

${ }^{8}$ Texas Children's Hospital, Houston, TX, USA.

${ }^{9}$ Department of Human Genetics, Radboud University Medical Center and Radboud Institute for Molecular Life Sciences, Nijmegen, The Netherlands.

${ }^{10}$ Children's Health Research Institute, London, ON, Canada.

${ }^{11}$ Department of Pediatrics, Schulich School of Medicine \& Dentistry, Western University, London,

ON, Canada.

${ }^{12}$ Division of Rheumatology, Children's Hospital, London, ON, Canada.

${ }^{13}$ Department of Medical Genetics, Rikshospitalet, Oslo University Hospital, Oslo, Norway.

${ }^{14}$ Department of Medical Genetics, Oslo University Hospital and University of Oslo, Oslo, Norway.

${ }^{15}$ Center for Rare Childhood Disorders, Translational Genomics Research Institute, Phoenix, AZ, USA.

${ }^{16}$ Baylor Genetics, Houston, TX, USA.

${ }^{17}$ AiLife Diagnostics, Pearland, TX, USA.

${ }^{18}$ Institute of Human Genetics, Heidelberg University, Heidelberg, Germany.

${ }^{19}$ These authors contributed equally to this work.

+ Current affiliations: Department of Clinical Genetics, University Medical Center Utrecht, Utrecht, The Netherlands; Princess Máxima Center for Pediatric Oncology, Utrecht, The Netherlands.

*Correspondence should be addressed to C. P. S. (schaaf@bcm.edu) or X.W. (xiaw@bcm.edu) 


\section{ABSTRACT}

Congenital heart defects and skeletal malformations syndrome (CHDSKM) is a rare autosomal dominant disorder characterized by congenital heart disease, skeletal abnormalities, and failure to thrive. CHDSKM is caused by germline mutations in ABL1. To date, three variants have been in association with CHDSKM. In this study, we describe three de novo missense variants, c.407C >T (p.Thr136Met), c.746C>T (p.Pro249Leu), and c.1573G >A (p.Val525Met), and one recurrent variant, c.1066G>A (p.Ala356Thr), in six patients, thereby expanding the phenotypic spectrum of CHDSKM to include hearing impairment, lipodystrophy-like features, renal hypoplasia, and distinct ocular abnormalities. Functional investigation of the three novel variants showed an increased ABL1 kinase activity. The cardiac findings in additional patients with p.Ala356Thr contribute to the accumulating evidence that patients carrying either one of the recurrent variants, p.Tyr245Cys and p.Ala356Thr, have a high incidence of cardiac abnormalities. The phenotypic expansion has implications for the clinical diagnosis of CHDSKM in patients with germline $A B L 1$ variants.

\section{KEYWORD}

CHDSKM, congenital heart defects, skeletal malformations, hearing impairment, renal hypoplasia 
Proto-oncogene $A B L 1$ is well known for being part of the fusion gene $B C R-A B L 1$ in the Philadelphia chromosome of leukemic cells(de Klein et al., 1982). Inherited ABL1 variants have only recently been reported in association with genetic disorders and germline heterozygous mutations in $A B L 1$ were found to cause congenital heart defects and skeletal malformations syndrome (CHDSKM; MIM\# 617602). Two germline missense variants, c.734A>G (p.Tyr245Cys) and c.1066G>A (p.Ala356Thr), were reported in patients with congenital heart disease, skeletal abnormalities, and failure to thrive. Some patients also displayed gastrointestinal problems, male genital/sexual abnormalities, and dysmorphic facial features. Although functional investigation of the two variants suggested an increased ABL1 kinase activity, none of the six reported patients in four unrelated families were reported to manifest malignancies(Wang et al., 2017).

Here we present three novel de novo missense variants, c.407C >T (p.Thr136Met), c.746C>T (p.Pro249Leu), and c.1573G>A (p.Val525Met), as well as one previously reported missense variant c.1066G>A (p.Ala356Thr) in ABL1 (NM_007313.2, isoform 1b) in a total of six patients sharing overlapping phenotypes. Additional clinical features, not previously reported in patients with $A B L 1$ variants, include hearing impairment, lipodystrophy-like features, hypoplastic kidney, and distinct ocular defects. In agreement with previously published information, functional investigation of the three novel missense variants showed an increased ABL1 kinase activity, indicating that the causative 
missense variants are likely gain-of-function mutations. This report expands the clinical spectrum of CHDSKM.

\section{$2 \quad$ Patient 1}

The patient was a 3-year-old female of Caucasian descent (Dutch, English, Scottish, and German). After birth, she was noted to have transient tachypnea and was discharged at 4 days of life. She was noted to have failure to thrive, severe feeding difficulties, axial hypotonia, and difficulties in balance. Skeletal abnormalities included mild clinodactyly and tapered fingers. While craniosynostosis or scoliosis were not found in the patient, she had plagiocephaly, torticollis, and early closure of anterior fontanelle. Gastrointestinal abnormalities included gastroesophageal reflux disease (GERD) and umbilical hernia. She had dysmorphic facial features, including mild facial asymmetry, triangular face, prominent forehead, frontal bossing, deep-set eyes, epicanthal folds, mildly short and upslanting palpebral fissures, wide nasal root, wide nasal bridge, small mouth, and micrognathia. There were no reported cardiac dysfunction; however, the sinus of Valsalva measured at upper limit of normal range at 2 years of age. Chromosome microarray analysis and metabolic workup were normal. Family history was significant for the mother having epilepsy, scoliosis, and unilateral deteriorating hip joint. The patient had two brothers, one with mild cognitive delay, severe astigmatism, and unilateral dilated renal pelvis and the other with severe asthma and autism. 
The patient was a 2-year-old Indian female. She was born at 36 weeks and 3 days gestational age via induced vaginal delivery with birth weight ( $2410 \mathrm{~g},<3^{\text {rd }}$ centile) and length $\left(47 \mathrm{~cm}, 13^{\text {th }}\right.$ centile). The pregnancy was complicated by placental calcification, placental insufficiency, marginal cord insertion, and intrauterine growth restriction (IUGR). A 34 weeks ultrasound showed frontal-occipital circumference (FOC) below $1^{\text {st }}$ centile. She had microcephaly, mild speech delay, mild hypotonia, feeding difficulties, and sleep disturbance. She had gastrointestinal abnormalities, including GERD, severe emesis, intestinal malrotation, delayed gastric emptying, umbilical hernia, and anteriorly placed anus. Her dysmorphic facial features included short palpebral fissures, low-hanging columella, downturned corners of mouth, and a high-arched palate. Echocardiogram and brain MRI were reported normal. Chromosome microarray analysis was normal. The FOC measurement at 2 years and 2 weeks of age was $44 \mathrm{~cm}\left(<3^{\text {rd }}\right.$ centile). Family history was significant for the mother and a maternal uncle with microcephaly. The FOC measurement of the mother was below $3^{\text {rd }}$ centile. While the mother did not have developmental delay, the maternal uncle had a history of delayed speech without learning problems.

\section{$4 \quad$ Patient 3}

The patient was a 30-year-old Dutch Caucasian male. He had delayed motor milestones and dyslexia. Skeletal abnormalities included long and slender fingers as well as thickening of the proximal interphalangeal joints of both hands with darkened and thin skin overlying these joints. The abnormalities of the proximal interphalangeal joints were suspected to 
be resulting from myofibroblastic proliferation, based on histological examination. Dysmorphic facial features included a long and narrow face, blepharophimosis, low-set ears, a narrow, elongated nose, short philtrum, small mouth, and hypoplasia of the maxilla. He had bilateral iris coloboma, hypodontia, mild vesicoureteral reflux, and a unilateral hypoplastic kidney. He had two pneumothoraces at 16 years of age. Cardiac ultrasound examination at 29 years of age was normal and showed no evidence of aortic root dilation. Chromosome microarray analysis, karyotyping, and telomere multiplex ligation-dependent probe amplification were normal.

\section{$5 \quad$ Patient 4}

The patient was a 20-year-old European Caucasian female. The pregnancy was complicated by IUGR. Her birth weight and birth length were $2620 \mathrm{~g}$ ( $8^{\text {th }}$ centile) and 46 $\mathrm{cm}\left(5^{\text {th }}\right.$ centile), with a head circumference $32 \mathrm{~cm}\left(6^{\text {th }}\right.$ centile). She had microcephaly, feeding difficulties, failure to thrive, mild speech delay, and short stature. Her skeletal abnormalities included bilateral camptodactyly of the $2^{\text {nd }}, 3^{\text {rd }}, 4^{\text {th }}$, and $5^{\text {th }}$ fingers, long tapering fingers, flexion contracture of the right elbow, overriding toes, pectus excavatum, and mild thoracic scoliosis. She had nodule formation in her hands, chronic thickening across the metatarsophalangeal joints, and tenosynovitis. Cardiac abnormalities included a congenital small secundum atrial septal defect with small shunt and mitral valve prolapse with mild mitral regurgitation. She had bilateral Eustachian tube dysfunction, hearing loss that required bilateral hearing aids, and bilateral nasolacrimal duct stenosis. She had a spontaneous pneumothorax, which was repaired by bullectomy 
with talc at 16 years of age. She had velopharyngeal insufficiency, bilateral cataracts, retinal detachment, fusion of teeth, and unusual fat distribution on her body, similar to lipodystrophy. She had tachypnea and dyspnea (mMRC Dyspnea Scale 2/4) which might be secondary to her cardiac condition or interstitial lung disease. She had cutis marmorata and velvety skin. She did not report gastrointestinal issues. Dysmorphic facial features included frontal bossing, bitemporal narrowing, a long and narrow face, deep set eyes, short palpebral fissures, epicanthal folds, small, low set, posteriorly rotated ears, broad nasal bridge, small mouth, micrognathia, and high-arched palate. At 19 years of age, her weight, length, and FOC were $37 \mathrm{~kg}\left(<3^{\text {rd }}\right.$ centile $), 156 \mathrm{~cm}\left(<10^{\text {th }}\right.$ centile $)$, and $51 \mathrm{~cm}\left(<3^{\text {rd }}\right.$ centile), respectively. She had normal mental development at 20 years of age. Chromosome microarray analysis, karyotyping, and sequencing of the PIK3R1 gene showed no detectable abnormalities.

\section{$6 \quad$ Patient 5}

The patient was an 11-year-old Norwegian male. The pregnancy was complicated by IUGR and the patient was born via induced vaginal delivery at 39 weeks gestation due to oligohydramnios and poor growth. His birth weight and birth length were $2307 \mathrm{~g}\left(<3^{\text {rd }}\right.$ centile) and $48 \mathrm{~cm}$ ( $10^{\text {th }}$ centile), with a FOC $32.5 \mathrm{~cm}\left(<3^{\text {rd }}\right.$ centile) based on Norwegian growth charts(Juliusson et al., 2009; Juliusson et al., 2013). He was born with scrotal hair. Brain MRI done one week after birth showed a thin corpus callosum, Dandy-Walker malformation, and hypoplasia of cerebellar vermis. The brain MRI has not been repeated ever since. He had global developmental delay, failure to thrive, hypotonia, and severe 
feeding difficulties requiring gastrostomy tube for insertion, tetralogy of Fallot, and microcephaly $\left(50.5 \mathrm{~cm} ;<3^{\text {rd }}\right.$ centile at 9 years of age based on Norwegian growth charts(Juliusson et al., 2009; Juliusson et al., 2013)). His skeletal abnormalities included camptodactyly, tapered fingers, pes planus, and overriding toes. He had lacrimal duct stenosis (surgery repaired at 2 years of age), recurrent ear infections, hearing impairment, severely crowded teeth, overbite, weak enamel, GERD, emesis, abnormal fat distribution around the waist, and accidental fecal and urinary incontinence at 9 years of age. The dysmorphic facial features included a long and narrow face, high forehead, bitemporal narrowing, short, upslanting palpebral fissures, epicanthal folds, high nasal bridge, low-set ears, a long nose with narrow alae nasi, small mouth, tented thin upper lip, high-arched palate, and prominent chin. He had cutis marmorata and velvety skin. He wore a positive expiratory pressure mask due to hypotonia of chest and abdominal wall musculature. He did not have intellectual disability at 11 years of age. Chromosome microarray analysis and karyotyping showed no detectable abnormalities.

\section{$7 \quad$ Patient 6}

The patient was a 5-year-old female of Hispanic descent. The pregnancy was complicated by IUGR. She was born at 39 weeks gestation with birth weight $2295 \mathrm{~g}\left(<3^{\text {rd }}\right.$ centile), birth length $49 \mathrm{~cm}\left(47^{\text {th }}\right.$ centile), and FOC $33 \mathrm{~cm}$ ( $23^{\text {rd }}$ centile). She had global developmental delay, failure to thrive, hypotonia, and feeding difficulties. Her skeletal abnormalities included joint hypermobility, pectus excavatum, $5^{\text {th }}$ finger clinodactyly, camptodactyly, long fingers with fetal fat pads, triphalangeal thumbs, pes planus, mild sandal gap, 
bilateral long $2^{\text {nd }}$ and $3^{\text {rd }}$ toes, overriding toes, talipes planovalgus, and congenital bilateral femoral anteversion. Cardiac abnormalities included atrial septal defects and mild aortic root dilation. Gastrointestinal abnormalities included GERD, emesis, constipation, intestinal malrotation, anteriorly placed anus, anal stenosis, and Morgagni hernia. She had dysmorphic facial features, including frontal bossing, arched and bushy eyebrows, long eyelashes, blepharophimosis, telecanthus, epicanthal folds, ptosis, depressed nasal bridge, bulbous nasal tip, smooth philtrum, small mouth, downturned corners of the mouth, thin upper lip vermilion, high-arched palate, small chin, and moderate micrognathia. MRI showed minimal proptosis of the left eye. She also had hearing loss that required left hearing aid, lacrimal duct stenosis, hypodontia, velvety skin, and chronic lung disease. Based on the manifesting clinical features, the differential diagnosis include Townes-Brocks syndrome. Chromosome microarray analysis showed no detectable abnormalities.

Written informed consent for all patients was obtained in accordance with protocols approved by the Institutional Review Board at Baylor College of Medicine. Proband-only or trio whole-exome sequencing was performed for all patients. Patient 1 was enrolled through BC Children's and BC Women's Hospitals, Vancouver, Canada. Patients 2 and 4 were enrolled through Baylor Genetics. Patient 3 was enrolled through the Radboud University Medical Center, Nijmegen, the Netherlands. Patient 5 was enrolled through Oslo University Hospital, Oslo, Norway. Patient 6 was enrolled through the Translational Genomics Research Institute, Phoenix, Arizona. All patient samples were analyzed by 
exome sequencing. The details of molecular analysis of each patient can be found in Supplementary information.

cDNA constructs, cell culture, and immunoblotting in the functional studies were performed as previously described(Wang et al., 2017). The anti-phospho-ABL1 antibody (\#5300, Cell Signaling Technology, Danvers, MA) used in the original study(Wang et al., 2017) was replaced with a different anti-phospho-ABL1 antibody (\#2861, Cell Signaling Technology, Danvers, MA) due to discontinuation of the antibody production. The methods of functional study in $A B L 1$ variants are provided in Supplementary information.

All reported heterozygous missense variants identified by exome sequencing in this study were found to be de novo (Supp. Figure S1 and Supp. Table S1 and S2). We identified the variant c.407C > T (p.Thr136Met) in patient 1; the variant c.746C >T (p.Pro249Leu) in patient 2; and two variants in cis, c.1573G>A (p.Val525Met) and c.1588G>C (p.Gly530Arg), in patient 3. A previously reported variant c.1066G $>$ A (p.Ala356Thr) was identified in patients 4,5 , and 6 . To evaluate the potential effects of the respective $A B L 1$ variants, we transiently transfected HEK 293T cells with plasmid constructs encoding C-terminally tagged wild-type and mutant proteins using human ABL1 cDNA (NM_007313.2). Overexpression of the mutant constructs resulted in increased overall phosphotyrosine levels and increased autophosphorylation of ABL1 itself when compared to cells expressing wild-type protein (Figure 1). These results suggested that the missense variants identified in this study lead to increased ABL1 kinase activity, except for the 
variant c.1588G >C (p.Gly530Arg) which was found in cis with c.1573G>A (p.Val525Met) in patient 3 (Supp. Figure S2). The variant c.1588G >C (p.Gly530Arg) does not increase ABL1 kinase activity in our experimental settings, suggesting that the c.1573G >A (p.Val525Met) variant is likely the cause of the disease in patient 3 . The facial, skeletal, and lipodystrophy-like features of the patients are shown in Figure 2. The comparison of clinical feature of the patients in this study versus the previous literature is shown in

\section{Supp. Table S3.}

This study reports six patients carrying de novo heterozygous missense variants with phenotypes overlapping with the original study(Wang et al., 2017). ABL1 has two isoforms, 1a (NM_005157.5) and 1b (NM_007313.2), as a result of an alternatively spliced first exon. Isoform $1 \mathrm{~b}$ has additional $\mathrm{N}$-terminal residues that are subject to myristoylation. Myristoylation of ABL1 in these residues has a role in autoinhibition of the kinase activity. The Thr136, Pro249, and Val525 residues are highly conserved amino acids. The Pro249 residue has been reported to be crucial for the autoinhibition of ABL1 kinase activity, which is consistent with the double mutation (p.Pro242Glu and p.Pro249Glu) resulting in higher kinase activity than that seen for wild-type protein(Pluk, Dorey, \& Superti-Furga, 2002). Similarly, the Val525 residue was shown to be critical for the regulation of ABL1 kinase activity, consistent with the p.Val525Asp mutation resulting in an increased kinase activity(Hantschel et al., 2003). A de novo variant p.Thr136Met has been reported in one female subject in a large cohort study looking into the contribution of de novo coding mutations to autism spectrum disorder(lossifov et al., 2014; Kosmicki et 
al., 2017). However, we were not able to compare that subject to patient 1 of this study, given the limited clinical information provided.

A de novo heterozygous in-frame deletion, c.434_436del (p.Ser145del) was recently reported in a patient with congenital heart defects, facial dysmorphism, skeletal malformations, and eye anomalies(Bravo-Gil, Marcos, Gonzalez-Meneses, Antinolo, \& Borrego, 2019). The authors suggested that p.Ser145del could lead to an increased ABL1 kinase activity based on the proposed interaction between Ser145 and Ser69 in ABL1 crystal structure as well as the increased kinase activity of the mutation p.Ser69Ala(Nagar et al., 2006). Although all $A B L 1$ missense variants reported to date show increased kinase activity $^{2}$, malignancies have not been reported in any of the affected patients.

The six patients in this study and the seven patients in the previous reports share common clinical features of CHDSKM, including skeletal and joint abnormalities (12/13), congenital heart disease (10/13), failure to thrive (10/13), gastrointestinal problems (10/13), and dysmorphic facial features (13/13) (Supp. Table S3). On the other hand, patients in this study have clinical features not reported previously, such as hearing impairment, lipodystrophy-like features, renal hypoplasia, and distinct ocular abnormalities (Figure 2, Supp. Table S3). Notably, minimal cardiac manifestations were found in patients 1-3 of this study, compared to patients carrying one of the recurrent variants, p.Tyr245Cys and p.Ala356Thr. This raises the question of whether the Tyr245 and Ala356 residues are more critical for the normal ABL1 function in cardiac development. Whether there is a possible 
genotype-phenotype correlation in CHDSKM will require the identification of additional patients and further investigation in the future.

Although cell culture experiment shows the $A B L 1$ variants in this study have increased kinase activity, which is consistent with previously reported $A B L 1$ variants underlying CHDSKM(Wang et al., 2017), it remained unclear whether the hyperactivity of ABL1 kinase directly leads to patient phenotypes, whether these variants still possess increased activity in intact organisms, or whether these variants result in the disruption of proteinprotein interaction between ABL1 and other proteins instead. It would be valuable to see whether knock-in mouse models bearing these missense variants actually recapitulate human phenotypes. In this study, patient 1 had a positive family history of neurodevelopmental disorder and skeletal abnormalities, while in patient 2 , the microcephaly was also seen in the mother and the maternal uncle. Given that the ABL1 variants in these two patients occurred de novo, the phenotypes seen in the family members were less likely attributing to the ABL1 variants. However, we were not able to completely rule out that certain overlapping clinical features shared by the proband and the family members may be confounded by environmental factors or other genetic variants not identified.

In summary, we expanded the clinical phenotypic spectrum associated with CHDSKM and suggested that the underlying germline $A B L 1$ missense variants are likely to be gain-of- 
function. Continued analyses of these variants and identification of additional patients are required to establish the causal relationship.

\section{ACKNOWLEDGEMENTS}

The authors sincerely thank all the family members for their participation in this study. This work was supported by the departmental fund from the Department of Molecular and Human Genetics at Baylor College of Medicine. The investigators of the CAUSES Study include Shelin Adam, Christele Du Souich, Alison Elliott, Anna Lehman, Jill Mwenifumbo, Tanya Nelson, Clara Van Karnebeek, and Jan Friedman. CAUSES Study is funded by Mining for Miracles, British Columbia Children's Hospital Foundation and Genome British Columbia. The investigators of the C4RCD Research Group include Chris Balak, Newell Belnap, Amanda Courtright-Lim, David Craig, Matthew Huentelman, Wayne Jepsen, Lori Llaci, Marcus Naymik, Ignazio Piras, Sampathkumar Rangasamy, Meredith SanchezCastillo, Szabolics Szelinger, and Morgan Wolfgang. The genome sequencing service of patient 5 was performed by the Norwegian High-Throughput Sequencing Centre, a national technology platform supported by the "Functional Genomics" and the "infrastructure" programs of the Research Council of Norway and the Southeastern Regional Health Authorities.

\section{CONFLICT OF INTEREST}

Y.Y. and X.W. holds stock ownership in AiLife Diagnostics. 


\section{AUTHOR CONTRIBUTIONS}

C.C. and E.C. performed the experiments. H.G., T. N. N., L. A. R., M. C. J. J., R. P., C. P., R. A.

B., M. F., E. F., D. M., and K. R. recruited $A B L 1$ patients and acquired clinical data. C.C., C.

P. S., and X. W. reviewed the genetic data and performed a critical revision of the manuscript for important intellectual content, while all other authors assisted in editing.

\section{DATA AVAILABILITY STATEMENT}

Variant data have been submitted to ClinVar (https://www.ncbi.nlm.nih.gov/clinvar/).

The data that support the findings of this study are available on request from the corresponding author. The data are not publicly available due to privacy restrictions. 


\section{REFERENCES}

Bravo-Gil, N., Marcos, I., Gonzalez-Meneses, A., Antinolo, G., \& Borrego, S. (2019). Expanding the clinical and mutational spectrum of germline ABL1 mutations-associated syndrome: A case report. Medicine (Baltimore), 98(10), e14782. doi:10.1097/MD.0000000000014782

de Klein, A., van Kessel, A. G., Grosveld, G., Bartram, C. R., Hagemeijer, A., Bootsma, D., . . Stephenson, J. R. (1982). A cellular oncogene is translocated to the Philadelphia chromosome in chronic myelocytic leukaemia. Nature, 300(5894), 765-767.

Hantschel, O., Nagar, B., Guettler, S., Kretzschmar, J., Dorey, K., Kuriyan, J., \& Superti-Furga, G. (2003). A myristoyl/phosphotyrosine switch regulates c-Abl. Cell, 112(6), 845-857. doi:10.1016/s00928674(03)00191-0

Iossifov, I., O'Roak, B. J., Sanders, S. J., Ronemus, M., Krumm, N., Levy, D., ... Wigler, M. (2014). The contribution of de novo coding mutations to autism spectrum disorder. Nature, 515(7526), 216221. doi:10.1038/nature13908

Juliusson, P. B., Roelants, M., Eide, G. E., Moster, D., Juul, A., Hauspie, R., . . Bjerknes, R. (2009). [Growth references for Norwegian children]. Tidsskr Nor Laegeforen, 129(4), 281-286. doi:10.4045/tidsskr.09.32473

Juliusson, P. B., Roelants, M., Nordal, E., Furevik, L., Eide, G. E., Moster, D., ... Bjerknes, R. (2013). Growth references for 0-19 year-old Norwegian children for length/height, weight, body mass index and head circumference. Ann Hum Biol, 40(3), 220-227. doi:10.3109/03014460.2012.759276

Kosmicki, J. A., Samocha, K. E., Howrigan, D. P., Sanders, S. J., Slowikowski, K., Lek, M., . . Daly, M. J. (2017). Refining the role of de novo protein-truncating variants in neurodevelopmental disorders by using population reference samples. Nat Genet, 49(4), 504-510. doi:10.1038/ng.3789

Nagar, B., Hantschel, O., Seeliger, M., Davies, J. M., Weis, W. I., Superti-Furga, G., \& Kuriyan, J. (2006). Organization of the SH3-SH2 unit in active and inactive forms of the c-Abl tyrosine kinase. $\mathrm{Mol}$ Cell, 21(6), 787-798. doi:10.1016/j.molcel.2006.01.035

Pluk, H., Dorey, K., \& Superti-Furga, G. (2002). Autoinhibition of c-Abl. Cell, 108(2), 247-259. doi:10.1016/s0092-8674(02)00623-2

Wang, X., Charng, W. L., Chen, C. A., Rosenfeld, J. A., Al Shamsi, A., Al-Gazali, L., . . Yang, Y. (2017). Germline mutations in ABL1 cause an autosomal dominant syndrome characterized by congenital heart defects and skeletal malformations. Nat Genet, 49(4), 613-617. doi:10.1038/ng.3815 
FIGURE LEGENDS

(a)

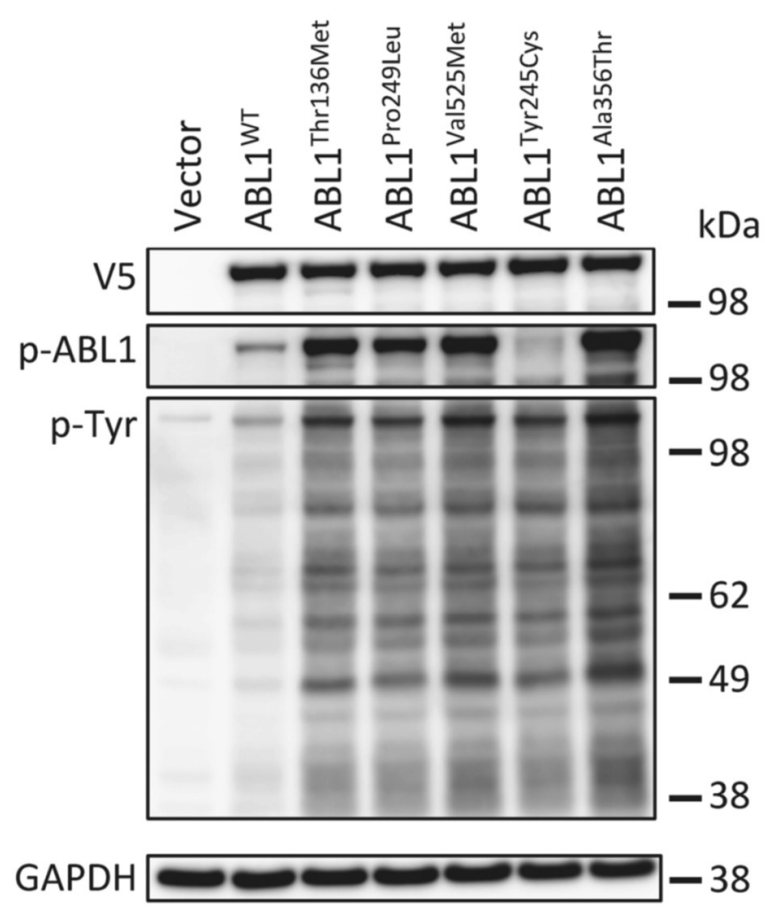

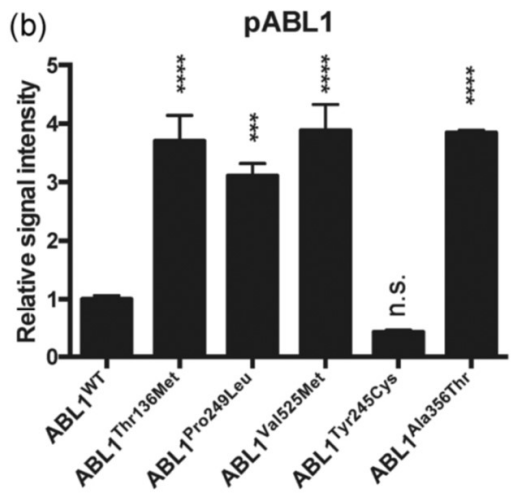

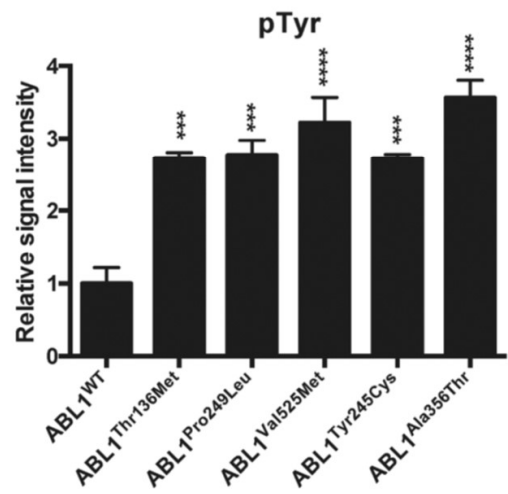

Figure 1. The effect of ABL1 variants on phosphorylation. (A) Overall phosphotyrosine levels and ABL1 auto-phosphorylation were analyzed by transiently expressing the wild-type and mutant constructs in HEK 293T cells and immunoblotting. Expression of all five variants resulted in increased overall phosphotyrosine levels when compared with cells expressing wild-type protein. Increased phosphorylated ABL1 was observed for all variants but not Tyr245Cys owing to the substitution of the Tyr245 residue, which is recognized by the anti-phospho-ABL1 antibody. The level of GAPDH was used as an internal loading control. Experiments for each construct were performed in triplicate. (B) Quantification of the immunoblot results. Data were normalized to GAPDH protein levels, with the wild-type protein set at 1.0. ${ }^{* * * P} \leq 0.001, * * * * P$ $\leq 0.0001$; n.s., $\mathrm{P}>0.05$, one-way ANOVA with Bonferroni multiple-comparisons test. 
(a)

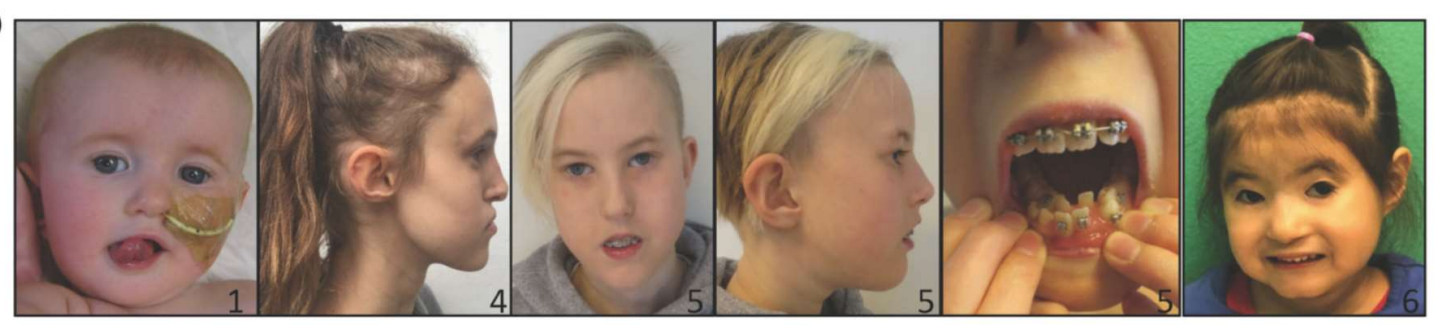

(b)

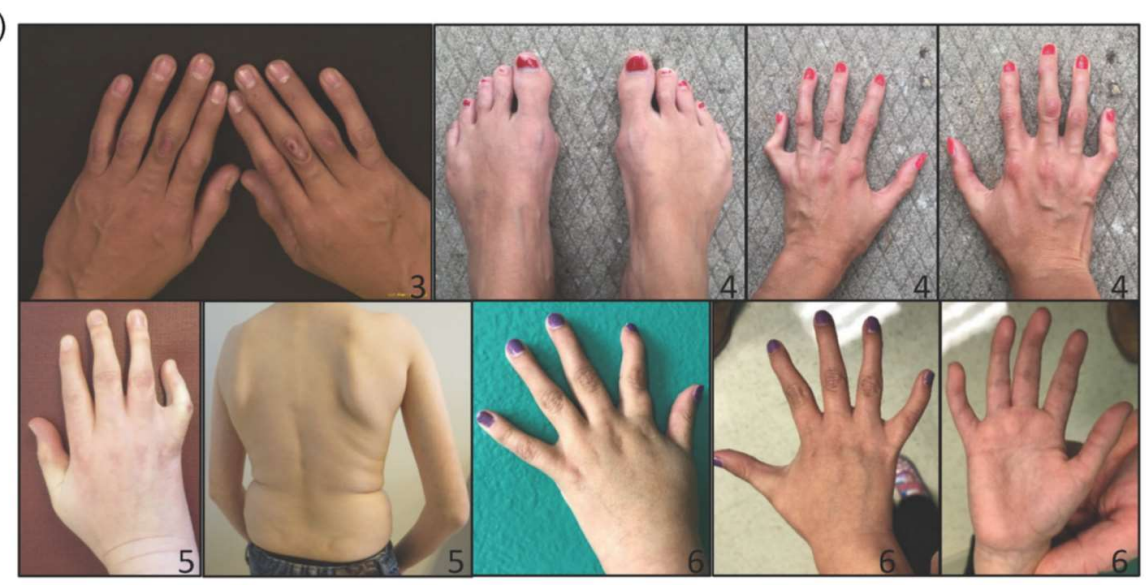

Figure 2. The facial, skeletal, and lipodystrophy-like features of patients $1,3,4,5$, and 6 . (A)

Facial features. From left to right: patients 1, 4, 5, and 6. Note long face with narrow maxilla and pointed chin in patients 4 and 5. (B) Skeletal and lipodystrophy-like abnormalities. Top from left to right: patients 3 and 4. Bottom from left to right: patients 5 and 6 . Abnormalities include flexion contractures, abnormal fat distribution around the waist, and triphalangeal thumb (patient 6). Informed consent was obtained from patients or guardians to publish these photographs. 


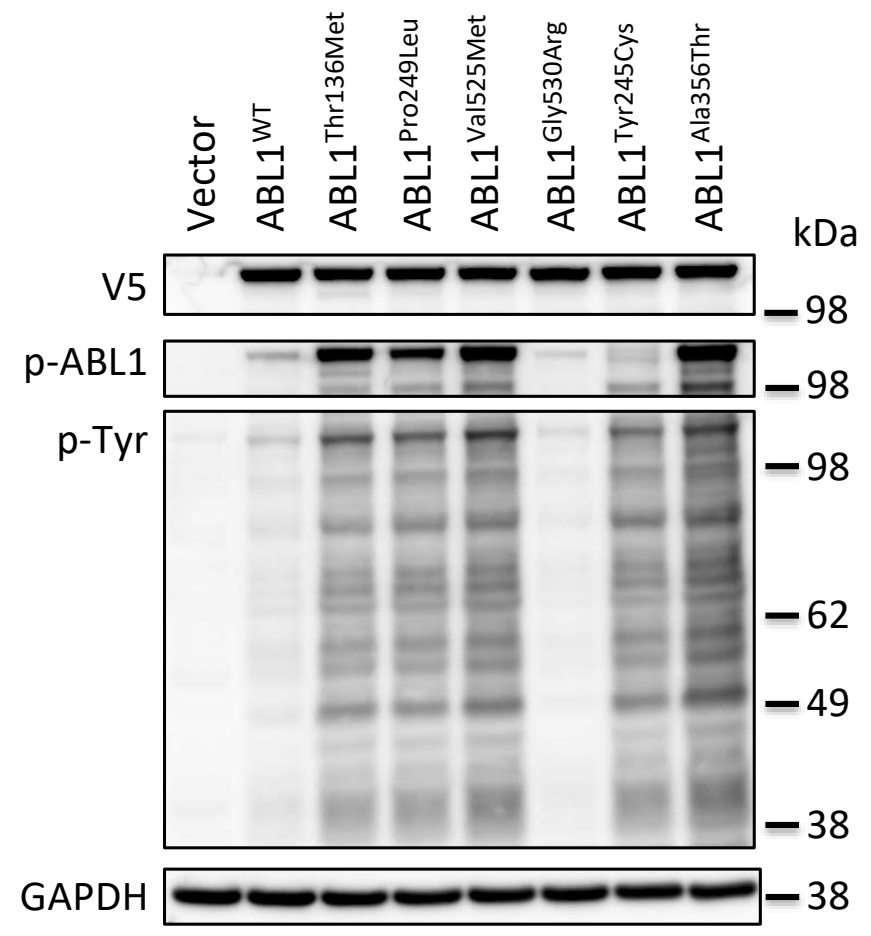

Supp. Figure S1. The effect of ABL1 variants on phosphorylation. Overall phosphotyrosine levels and ABL1 auto-phosphorylation were analyzed by transiently expressing the wild-type and mutant constructs in HEK 293T cells and immunoblotting. 


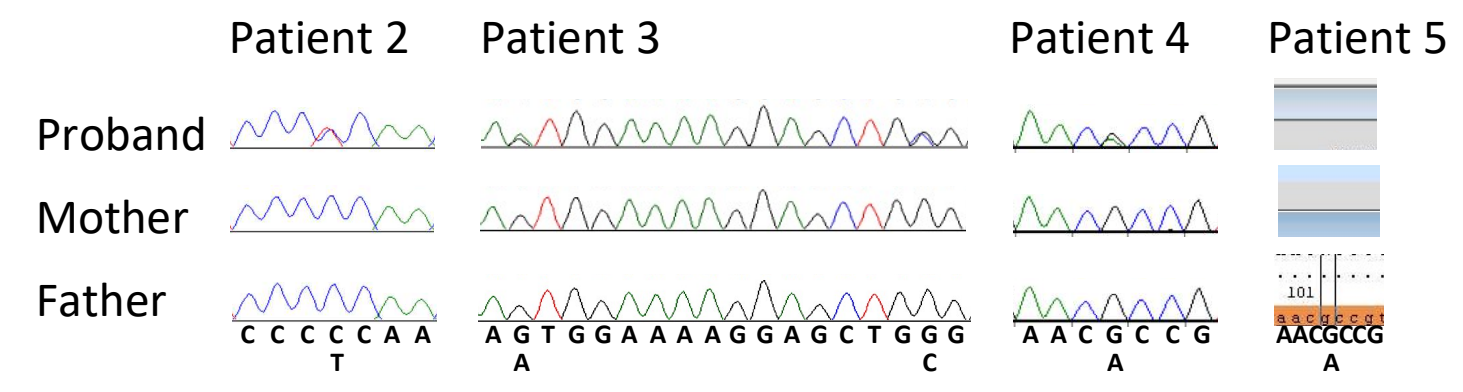

Supp. Figure S2. The Sanger sequencing of the $A B L 1$ variants in patients. The c.746C $>T$ (p.Pro249Leu) variant was identified in patient 2 . The two variants, c.1573G>A (p.Val525Met) and c.1588G>C (p.Gly530Arg), were identified in patient 3. Sequencing reads encompassing both variants through exome sequencing in patient 3 indicated that the two changes are located on the same chromosome (data not shown). The c.1066G>A (p.Ala356Thr) variant was identified in patients 4 and patient 5 . The Sanger sequencing results of patient 1 and patient 6, both of whom underwent trio whole genome/exome sequencing, were not available. 
Supp. Table S1: Annotation of the reported ABL1 variants

\begin{tabular}{|c|c|c|c|c|c|c|c|c|c|c|c|c|c|}
\hline 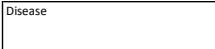 & $\begin{array}{l}\text { Inheritance } \\
\text { pattern }\end{array}$ & Gene & Position & Isoform & Location & Nucleotide & Amino acid & Zygosity & $\begin{array}{l}\text { In silico predictions } \\
\text { Sift } 7 \text { /PolyPhen-2 }\end{array}$ & ClinVar & $\begin{array}{l}\text { gnomad } \\
\text { Total allele } \\
\text { cuntt }\end{array}$ & Inheritance & $\begin{array}{l}\text { Pathogenicity classification by } \\
\text { ACMG guidelines (category) }\end{array}$ \\
\hline $\begin{array}{l}\text { Congenital heart defects and skeletal } \\
\text { malformations syndrome } \\
\text { (CHDSKM; MIM\# 617602) }\end{array}$ & AD & $A B L 1$ & \begin{tabular}{|l} 
chr9:133730284C>T \\
(hg19)
\end{tabular} & NM_007313.2 & Exon 3 & c.407 \T & p.Thr136Met & Het & \begin{tabular}{|l} 
Damaging/ \\
Probably \\
damaging
\end{tabular} & N/A & N/A & de novo & Pathogenic (PS2, PS3, PM2, PP3) \\
\hline \begin{tabular}{|l} 
Congenital heart defects and skeletal \\
malformations syndrome \\
(CHDSKM; MIM\# 617602$)$
\end{tabular} & $A D$ & $A B L 1$ & \begin{tabular}{|l} 
chrg:133738289C>T \\
(hg19)
\end{tabular} & NM_007313.2 & Exon 4 & $c .746 C>\mathrm{T}$ & \begin{tabular}{l|l|l} 
p.Pro249leu & \\
\end{tabular} & Het & \begin{tabular}{|l} 
Damaging/ \\
Probably \\
damaging
\end{tabular} & N/A & $\mathrm{N} / \mathrm{A}$ & de novo & $\begin{array}{l}\text { Likely pathogenic (PS3, PM2, PM6, } \\
\text { PP3) }\end{array}$ \\
\hline $\begin{array}{l}\text { Congenitat heart defects and skeletal } \\
\text { malformations syndrome } \\
\text { (CHDSKM; MIM\# 617602) }\end{array}$ & AD & ABL1 & \begin{tabular}{|l} 
chr9:133755889G>A \\
(hg19)
\end{tabular} & NM_007313.2. & Exon 10 & $c .1573 G>A$ & \begin{tabular}{l|l} 
p.Val525Met \\
\end{tabular} & Het & \begin{tabular}{|l} 
Damaginin/ \\
Probably \\
damaging
\end{tabular} & N/A & N/A & de novo & Pathogenic (PS2, PS3, PM2, PP3) \\
\hline \begin{tabular}{|l} 
Congenital heart defects and skeletal \\
malformations syndrome \\
(CHDSKM; MIM\# 617602$)$
\end{tabular} & $A D$ & $A B L 1$ & 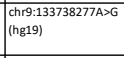 & NM_007313.2 & Exon 4 & $c .734 A>G$ & \begin{tabular}{|l|l} 
p.Tyr245Cys \\
\end{tabular} & Het & \begin{tabular}{|l}
$\begin{array}{l}\text { Damaging/ } \\
\text { Probably } \\
\text { damaging }\end{array}$ \\
\end{tabular} & Pathogenic & $\mathrm{N} / \mathrm{A}$ & \begin{tabular}{|l|} 
de novo/ \\
familial co- \\
segregation
\end{tabular} & \begin{tabular}{|l|}
$\begin{array}{l}\text { Pathogenic (PS2, PS3, PM2, PP1, } \\
\text { PP33) }\end{array}$ \\
\end{tabular} \\
\hline \begin{tabular}{|l} 
Congenital heart defects and skeletal \\
malformations syndrome \\
(CHDSKM; MMM\# 617602$)$ \\
\end{tabular} & $\mathrm{AD}$ & $A B L 1$ & $\begin{array}{l}\text { chrg: } 1337483486 \times A \\
\text { (hg19) }\end{array}$ & NM_007313.2 & Exon 6 & $c .1066 \mathrm{G}>\mathrm{A}$ & p.Ala356Thr & Het & \begin{tabular}{|l|}
$\begin{array}{l}\text { Damaging/ } \\
\text { Probably } \\
\text { damaging }\end{array}$ \\
\end{tabular} & Pathogenic & $\mathrm{N} / \mathrm{A}$ & de novo & $\begin{array}{l}\text { Pathogenic (PS2, PS3, PM1, PM2, } \\
\text { PP3) }\end{array}$ \\
\hline
\end{tabular}

Supp. Table S2: Exome sequencing identified variants in known disease genes related to the patient's phenotypes but unlikely to cause disease

\begin{tabular}{|c|c|c|c|c|c|c|c|c|c|c|c|c|}
\hline \multicolumn{13}{|c|}{ Patient 2} \\
\hline \begin{tabular}{|l} 
Disease \\
\end{tabular} & $\begin{array}{l}\text { Inheritance } \\
\text { pattern }\end{array}$ & Gene & Position & Isoform & Location & \begin{tabular}{|l} 
Nucleotide \\
\end{tabular} & Amino acid & Zygosity & SIFT/PolyPhen-2 & ClinVar & \begin{tabular}{|l}
$\begin{array}{l}\text { gnomAD } \\
\text { Total allele } \\
\text { count }\end{array}$ \\
\end{tabular} & Inheritance \\
\hline \begin{tabular}{|l|} 
Ciliary dyskinesia, primary, 14 \\
(CILD14; MIM\#\# 613807)
\end{tabular} & AR & $C C D C 39$ & $\begin{array}{l}\text { chr3:180372581A>G } \\
\text { (hb19) }\end{array}$ & NM_181426.2 & Exon 7 & $c .899 A>G$ & p. His300Arg & Het & $\begin{array}{l}\text { Tolerated/ } \\
\text { Benign }\end{array}$ & N/A & 5 & $\mathrm{~N} / \mathrm{A}$ \\
\hline \begin{tabular}{|l} 
Ciliary dyskinesia, primary, 15 \\
(CILD15; MIM\# 613808)
\end{tabular} & AR & $C C D C 40$ & $\begin{array}{l}\text { chr177:78073329C>G } \\
\text { (hg19) }\end{array}$ & NM_017950.4 & $\begin{array}{l}\text { Exon20 (last } \\
\text { exon) }\end{array}$ & $c .3184 \mathrm{C}>\mathrm{G}$ & p.Leu1062Val & Het & \begin{tabular}{|l|} 
Damaging/ \\
Possibly \\
damaging
\end{tabular} & N/A & 34 & N/A \\
\hline \begin{tabular}{|l} 
Ciliary dyskinesia, primary, 29 \\
(CILD29; MIM\# 615872)
\end{tabular} & AR & CCNO & \begin{tabular}{|l} 
chr5::54528984C>T \\
(hg19)
\end{tabular} & NM_021147.5 & Exon 1 & $c .368 \subset \mathrm{T}$ & \begin{tabular}{|l|} 
p.Ala123Val \\
\end{tabular} & Het & $\begin{array}{l}\text { Tolerated/ } \\
\text { Probably } \\
\text { damaging }\end{array}$ & N/A & 1 & N/A \\
\hline
\end{tabular}

\begin{tabular}{|c|c|c|c|c|c|c|c|c|c|c|c|c|}
\hline \multicolumn{13}{|c|}{ Patient 3} \\
\hline Disease & $\begin{array}{l}\text { Inheritance } \\
\text { pattern }\end{array}$ & Gene & Position & Isoform & \begin{tabular}{|l|l|} 
Location \\
\end{tabular} & Nucleotide & Amino acid & \begin{tabular}{|l|} 
Zygosity \\
\end{tabular} & SIFT/PolyPhen-2 & ClinVar & \begin{tabular}{|l} 
gnomAD \\
Total allele \\
count
\end{tabular} & Inheritance \\
\hline $\begin{array}{l}\text { Congenital heart defects and skeletal } \\
\text { malformations syndrome } \\
\text { (CHDSKM; MIM\# 617602) }\end{array}$ & AD & ABL1 & $\begin{array}{l}\text { chrg:133755904G>A } \\
\text { (hg19) }\end{array}$ & NM_007313.2 & Exon 10 & c. $1588 \mathrm{G}>\mathrm{C}$ & p.Gly530Arg & Het & \begin{tabular}{|l|} 
Damaging/ \\
Possibly \\
damaging
\end{tabular} & N/A & N/A & de novo \\
\hline
\end{tabular}

\begin{tabular}{|c|c|c|c|c|c|c|c|c|c|c|c|c|}
\hline \multicolumn{13}{|c|}{ Patient 6 } \\
\hline Disease & \begin{tabular}{|l} 
Inheritance \\
pattern
\end{tabular} & Gene & Position & | Isoform & Location & | Nucleotide & Amino acid & Zygosity & |SIFT/PolyPhen-2 & ClinVar & \begin{tabular}{|l} 
gnomAD \\
Total allele \\
count
\end{tabular} & Inheritance \\
\hline \begin{tabular}{|l} 
Central core disease \\
(CCD; MIIM\# 117000) \\
\{Malignant hyperthermia susceptibility 1\} \\
(MHS1; MIM\# 145600) \\
MHinicore myopathy with external \\
ophthalmoplegia \\
(MIM\# 255320)
\end{tabular} & $A D, A R$ & RYR1 & $\begin{array}{l}\begin{array}{l}\text { chr19:38956793C>T } \\
\text { (hg19) }\end{array} \\
\text { (hg) }\end{array}$ & NM_000540.3 & Exon 24 & c.2933C>T & \begin{tabular}{|l|} 
p.Pro978Leu \\
\end{tabular} & Het & \begin{tabular}{|l|} 
Damaging/ \\
Possibly \\
damaging
\end{tabular} & \begin{tabular}{|l} 
Likely \\
benign/ \\
Uncertain \\
significance
\end{tabular} & 74 & maternal \\
\hline \begin{tabular}{|l|} 
Central core disease \\
(CCD; MIM\# 117000) \\
\{Malignant hyperthermia susceptibility 1\} \\
(MHS1; MIM\# 145600) \\
MHinicore myopathy with external \\
ophthalmoplegia \\
(MIM\# 255320) \\
\end{tabular} & $A D, A R$ & RYR1 & $\begin{array}{l}\text { chr19:38993204G>A } \\
\text { (hg19) }\end{array}$ & NM_000540.3 & Exon 48 & $c .7672 G>A$ & p.Val2558Met & Het & \begin{tabular}{|l|} 
Damaging/ \\
Possibly \\
damaging
\end{tabular} & \begin{tabular}{|l|}
$\begin{array}{l}\text { Uncertain } \\
\text { significance }\end{array}$ \\
\end{tabular} & 30 & paternal \\
\hline
\end{tabular}


Supp. Table S3: Clinical and genetic findings in the affected individuals

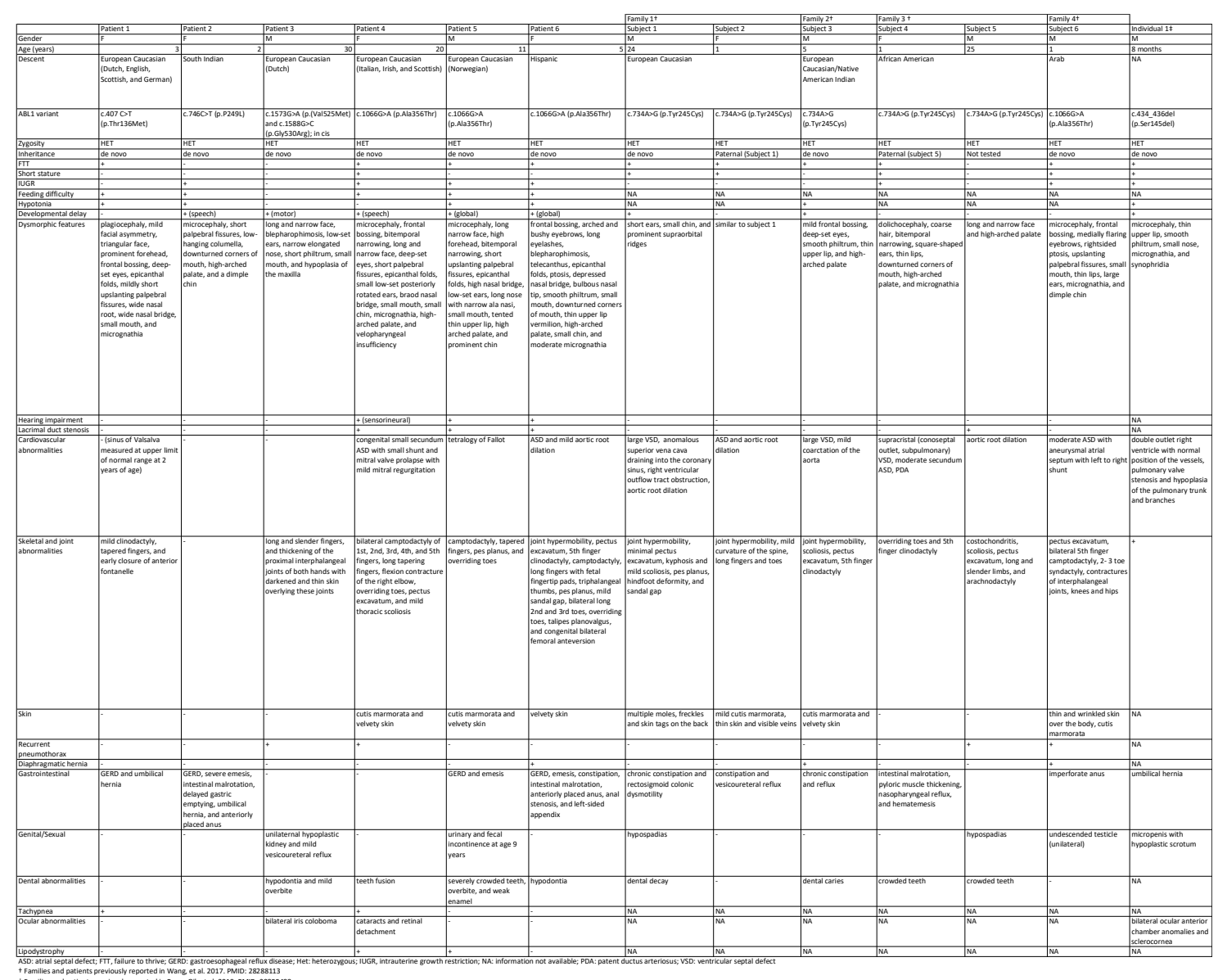




\section{Molecular analysis}

Patient 1:

Whole-genome sequence data generation of proband and parental samples with at least $98 \%$ of CCDS exons having at least 10X read depth coverage was performed at Canada's Michael Smith Genome Sciences Centre (British Columbia, Canada) through a translational research study (CAUSES Study), with institutional ethical approval from the University of British Columbia Columbia [H15-00092]. Read alignment and variant calling was performed with BWAMEM(1 1$)$ and GATK HaplotypeCaller(2). Variants were annotated and filtered with VarSeq ${ }^{\mathrm{TM}}$ v1.4.5, Golden Helix, Inc., Bozeman, MT and prioritized through multi-disciplinary assessment. Clinical Sanger sequencing of the proband and parental samples confirmed the de novo $A B L 1$ variant.

Patient 2:

Exome sequencing was performed as previously described(3). Briefly, genomic DNA samples were fragmented, ligated to Illumina multiplexing paired-end adapters, amplified with indexes added, and hybridized to a solution based exome capture reagent (Roche NimbleGen). Paired-end sequencing (100 $\mathrm{bp} \times 2$ ) was performed on Illumina HiSeq 2500 platform to provide a mean sequence coverage of about $120 \times$, with about $97 \%$ of the target bases having at least $20 \times$ coverage. Exome sequencing data was processed and the variants were annotated as previously described( $\underline{3})$. Briefly, the output data from the Illumina HiSeq 2500 were converted from a bcl file to a FastQ file by Illumina Consensus Assessment of Sequence and Variation software version 1.8.3, and mapped to the human-genome reference using

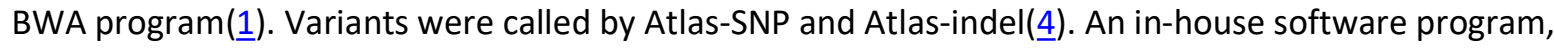


CASSANDRA, was used for variant filtering and annotation(그). Sanger sequencing of the parental samples confirmed the de novo ABL1 variant.

Patient 3:

Whole exome sequencing analysis was performed in a trio diagnostic approach (patient and both parents) at the Radboud University Medical Center in Nijmegen, the Netherlands, essentially as described before(므). Exome capture was performed with the Agilent SureSelect Human All Exon v5 enrichment kit (Agilent Technologies). Whole-exome sequencing was performed on the Illumina HiSeq platform (BGI, Copenhagen, Denmark). Data were analysed with BWA (read alignment,) and GATK (variant calling) software packages. Variants were annotated using an in-house developed pipeline. Prioritization of variants was done by an in-house designed 'variant interface' and manual curation. Sanger sequencing of the parental samples confirmed the de novo $A B L 1$ variant.

Patient 4:

Proband-Whole exome sequencing analysis was performed at GeneDx for clinical diagnostic purposes. DNA was sequenced on the Illumina HiSeq platform with $100 \mathrm{bp}$ or greater paired-end reads and captured with the Agilent Sure Select Kit. Reads were aligned to reference sequence GRCh37/UCSC hg19 and analyzed for variants using a custom-developed analysis tool (Xome Analyzer). Reporting was based on clinical information provided. Sanger sequencing of the parental samples confirmed the de novo $A B L 1$ variant. 
Patient 5:

Genomic DNA from the proband and both parents were used to capture exomes with the Illumina TruSeq Exome Enrichment kit and sequenced on an Illumina HiSeq2000 with 100 bp paired-end reads. Alignment against the GRCh37 human reference genome was performed with a Burrows-Wheeler Aligner (BWA, v.0.5.9)(11). PCR-duplicates marking and removal with Picard (v.1.104), and indel realignment, base quality recalibration, and joint variant calling with the Genome Analysis Toolkit (GATK, v.2.5)(ㅇ). Finally, functional annotation was performed with snpEff (v.2.05)(ㄱ) and Variant Effect Predictor (VEP)( $\underline{8})$ using Ensembl 71. The genetic variants were filtered using the program FILTUS v.99.91(9). Variants were removed if they had a high probability of being technical artifacts, as computed by the GATKs "variant quality score recalibration" procedure, occurred with a frequency higher than 0.01 in the 1000 Genomes Project (http://browser.1000genomes.org), the NHLBI Exome Sequencing Project Exome Variant Server (http://evs.gs.washington.edu/EVS), or in our in-house database of 440 exomes, or were predicted to have low impact according to snpEff(그), SIFT( $\underline{10})$ or PolyPhen-2(11). Sanger sequencing confirmed the de novo $A B L 1$ variant.

Patient 6:

Genomic DNA was extracted from blood taken from the proband and both parents. Whole-exome sequencing was performed at the Translational Genomics Research Institute. Exomic libraries were prepared with the Illumina TruSeq Exome Enrichment Kit v2. Sequencing was performed by $125 \mathrm{bp}$ paired-end sequencing on a HiSeq2000 instrument (Illumina Inc., San Diego, CA). Filtered reads were aligned to the human genome (Hg19/GRC37) using the BurrowsWheeler transform. Reads were sorted, and PCR duplicates were removed using Picard, and base-quality recalibration and indel realignment were performed using the Genome Analysis Toolkit. Variants were called jointly with haplotype caller and annotated with dbNSFP and SnpEff for protein-coding events. Prediction scores were loaded from 
$\mathrm{dbNSFP}$ and used for filtering. An annotated variant file containing variants in 3 family members was filtered to include novel, private, or rare variants according to the Exome Aggregation Consortium (ExAC) database and the Genome Aggregation Database (gnomAD). Variants predicted to be damaging by the Combined Annotation Dependent Depletion (CADD) tool or to disrupt protein structure or function by Polymorphism Phenotyping v2 (PolyPhen2) algorithms were considered as candidate genes responsible for the proband's phenotype. Sanger sequencing confirmed the de novo ABL1 variant.

\section{Functional study of $A B L 1$ variants}

ABL1 1b isoform cDNA clone (MHS6278-211687872) was purchased from GE Dharmacon (MGC cDNA collection). All point mutations were generated by QuikChange site-directed mutagenesis kit (Agilent). $A B L 1$ cDNA constructs were cloned into pcDNA ${ }^{\mathrm{TM}} 3.1 / \mathrm{V} 5$ - His A (with C-terminal V5 and His tags; Clontech) using Kpnl and Xhol restriction enzyme sites.

HEK 293T cell line was cultured in Dulbecco's Modified Eagle Medium (Corning 45000-312) with 10\% fetal bovine serum, L-glutamine, and antibiotics-antimycotics at $37{ }^{\circ} \mathrm{C}$ under $5 \% \mathrm{CO} 2$. The transfection was performed using Lipofectamine 2000 (ThermoFisher Scientific) according to the manufacturer's instructions. Cells were harvested $24 \mathrm{hrs}$ after transfection. Cells were lysed in lysis buffer [ $50 \mathrm{mM}$ Tris$\mathrm{HCl} \mathrm{pH} \mathrm{7.5,} 150 \mathrm{mM} \mathrm{NaCl}, 5 \%$ glycerol, 0.5\% TritonX-100, protease inhibitor cocktail and phosphatase inhibitor Xpert (Gendepot)]. The lysate supernatant was boiled in Laemmli buffer. Polyacrylamide gel electrophoresis, transfer, and Western blot were performed according to standard protocols. Primary antibodies were used at the following dilution: mouse $\alpha$-phospho Tyr 4G10 1:20,000 (EMD Millipore 05321), rabbit $\alpha$-phospho-c-Abl (Tyr245) 1:1,000 (Cell signaling 2861), mouse $\alpha$-V5 1:10,000 (ThermoFisher Scientific 460705), and mouse $\alpha$-GAPDH 1:20,000 (Advanced ImmunoChemical, 2-RGM2). Goat anti rabbit HRP-conjugated secondary antibody (Bio-rad 170-5046) was used at 1:10,000, and Donkey anti 
mouse HRP-conjugated secondary antibody (Jackson ImmunoResearch 715-035-150) was used at

$1: 10,000$.

\section{References}

1 Li, H. and Durbin, R. (2010) Fast and accurate long-read alignment with Burrows-Wheeler transform. Bioinformatics, 26, 589-595.

2 McKenna, A., Hanna, M., Banks, E., Sivachenko, A., Cibulskis, K., Kernytsky, A., Garimella, K., Altshuler, D., Gabriel, S., Daly, M. et al. (2010) The Genome Analysis Toolkit: a MapReduce framework for analyzing next-generation DNA sequencing data. Genome Res, 20, 1297-1303.

3 Yang, Y., Muzny, D.M., Reid, J.G., Bainbridge, M.N., Willis, A., Ward, P.A., Braxton, A., Beuten, J., Xia, F., Niu, Z. et al. (2013) Clinical whole-exome sequencing for the diagnosis of mendelian disorders. $N$ Engl J Med, 369, 1502-1511.

4 Shen, Y., Wan, Z., Coarfa, C., Drabek, R., Chen, L., Ostrowski, E.A., Liu, Y., Weinstock, G.M., Wheeler, D.A., Gibbs, R.A. et al. (2010) A SNP discovery method to assess variant allele probability from next-generation resequencing data. Genome Res, 20, 273-280.

$5 \quad$ Neveling, K., Feenstra, I., Gilissen, C., Hoefsloot, L.H., Kamsteeg, E.J., Mensenkamp, A.R., Rodenburg, R.J., Yntema, H.G., Spruijt, L., Vermeer, S. et al. (2013) A post-hoc comparison of the utility of sanger sequencing and exome sequencing for the diagnosis of heterogeneous diseases. Hum Mutat, 34, 1721-1726.

6 DePristo, M.A., Banks, E., Poplin, R., Garimella, K.V., Maguire, J.R., Hartl, C., Philippakis, A.A., del Angel, G., Rivas, M.A., Hanna, M. et al. (2011) A framework for variation discovery and genotyping using next-generation DNA sequencing data. Nat Genet, 43, 491-498.

$7 \quad$ Cingolani, P., Platts, A., Wang le, L., Coon, M., Nguyen, T., Wang, L., Land, S.J., Lu, X. and Ruden, D.M. (2012) A program for annotating and predicting the effects of single nucleotide polymorphisms, SnpEff: SNPs in the genome of Drosophila melanogaster strain w1118; iso-2; iso-3. Fly (Austin), 6, 80-92.

8 McLaren, W., Pritchard, B., Rios, D., Chen, Y., Flicek, P. and Cunningham, F. (2010) Deriving the consequences of genomic variants with the Ensembl API and SNP Effect Predictor.

Bioinformatics, 26, 2069-2070.

9 Vigeland, M.D., Gjotterud, K.S. and Selmer, K.K. (2016) FILTUS: a desktop GUI for fast and efficient detection of disease-causing variants, including a novel autozygosity detector.

Bioinformatics, 32, 1592-1594.

10 Kumar, P., Henikoff, S. and Ng, P.C. (2009) Predicting the effects of coding non-synonymous variants on protein function using the SIFT algorithm. Nat Protoc, 4, 1073-1081.

11 Adzhubei, I.A., Schmidt, S., Peshkin, L., Ramensky, V.E., Gerasimova, A., Bork, P., Kondrashov, A.S. and Sunyaev, S.R. (2010) A method and server for predicting damaging missense mutations. Nat Methods, 7, 248-249. 